\title{
Study of Metallic Dislocations by Methods of Nondestructive Evaluation Using Eddy Currents
}

\author{
Bettaieb Laroussi, Kokabi Hamid and Poloujadoff Michel \\ Université Pierre et Marie Curie (UPMC), Laboratoire d'Electronique et \\ Electromagnétisme (L2E), Paris
}

France

\section{Introduction}

This chapter summarizes a work which we have conducted over the period 2005-2010 [Bettaieb et al., 2008; Bettaieb, 2009]. The aim was to better understand the Non Destructive Evaluation (NDE) method based on the use of eddy currents. A very simple example, well adapted for beginners, was the evaluation of a homogenous rectangular aluminum plate of constant thickness, in case of a calibrated crack. Such a choice had a great advantage: it allowed the development of simple theoretical analysis, and therefore a very good understanding of the physics of the problem.

Naturally, theoretical results must be checked experimentally. To this end, we built a Helmholtz arrangement of two circular coils, to create a convenient uniform alternating field within the plate. Then, to measure the reaction field of the plate, we had to use a sensor. We used alternately a SQUID and a Hall sensor. The choice between those is not obvious, as will be shown later. Indeed, the SQUID is about 1000 times more sensitive than the Hall sensor, but its sensitive part is more remote than the Hall sensor, and this may reduce significantly its advantage. We found this comparison extremely interesting [Bettaieb et al., 2010].

Indeed, if we cut a rectangular plate, the cut being parallel to one side and normal to the excitation field, it seems natural that the plate is equivalent to a homogeneous one. Therefore, the cut cannot be detected. Just in case, we made the experiment and there was an unexpected signal!! We rapidly realized the reason of this result: the cut through the plate did not interrupt any current, but the cutting tool had damaged the microstructure of the aluminum over a small part of each half plate. Therefore, none of the two half parts had remained homogeneous. We realized that the microstructure of a small part of the half plates had been modified. In fact, the two halves of the original plate were equivalent to a homogeneous plate, except along the cut. Therefore, we did not detect the cut, but the metallic dislocations around it. Some more theoretical investigations allowed to evaluate the importance of the change in the electrical resistivity associated with the dislocations, and their extension. Then, we started to investigate the dislocations due to hammer shocks and to mechanical flexions. 
This chapter is a report of all our efforts. And we hope that other studies, by other investigators, will extend these results.

\section{Theoretical study of a defect free rectangular aluminium plate}

We consider the case of a homogeneous rectangular aluminum plate (resistivity $\rho=5.82 \times 10^{-8} \Omega . m$ ). Its dimensions are $a=110 \mathrm{~mm}, b=100 \mathrm{~mm}, c=5 \mathrm{~mm}$ in the $x, y, z$ directions respectively (figure 1). This plate is immersed in an alternative uniform magnetic induction field $\vec{B}$ parallel to the $O y$ direction, varying at the $180 \mathrm{~Hz}$ frequency, with an amplitude of $0.15 \times 10^{-4} \mathrm{~T}$ if we use a SQUID and $2.45 \times 10^{-4} \mathrm{~T}$ if we use a Hall probe sensor.

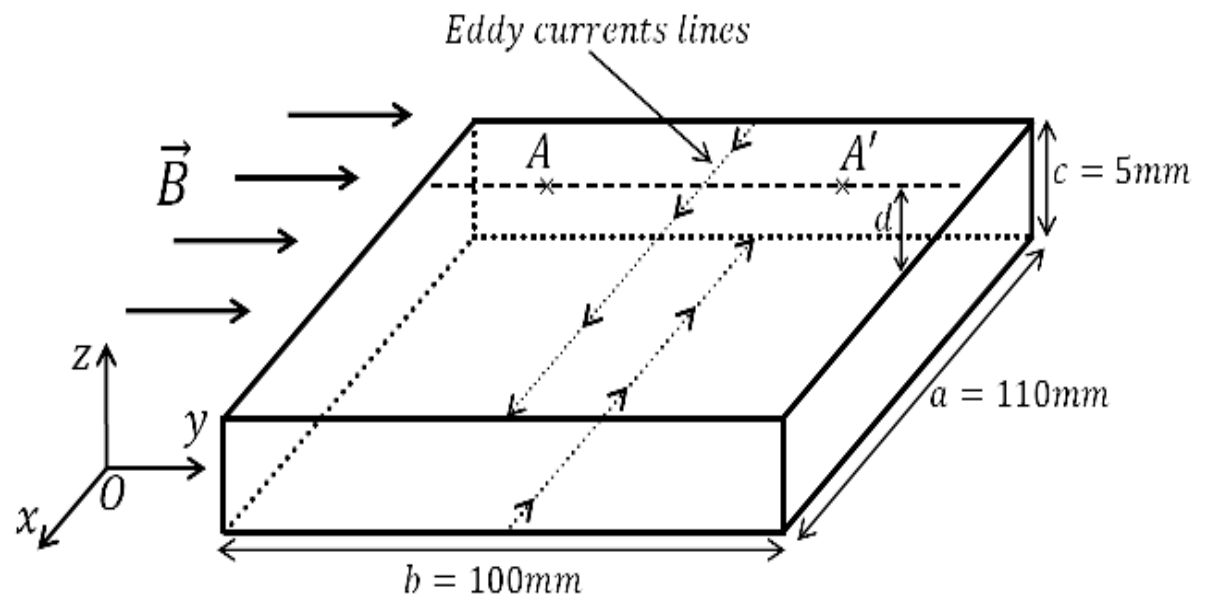

Fig. 1. Defect free rectangular aluminium plate under consideration

This excitation frequency has been chosen because it is one of those which are the less present in the general environment in and around our laboratory.

We shall study the induced current density using the more appropriate simplifying assumptions, in order to develop a good physical understanding of the phenomena.

In a first step, we assume that all the induced currents lines are straight lines parallel to the $O x$ direction from one side to the other (figure 1). If this is so, the external field is easy to evaluate by Biot and Savart law. An interesting remark is that the field of the induced currents, inside the plate, is quite much smaller than the excitation field.

In a second step, we improve the evaluation of the induced current lines in a plane abcd parallel to the plane $\mathrm{xOz}_{\mathrm{z}}$ (figure 2).

In such planes, the value of $\vec{B}$ is given under the form of a double Fourier expansion

$$
B(x, z)=\sum_{m, n} a_{m n} \cos \left(\frac{m \pi x}{a}\right) \cos \left(\frac{n \pi z}{c}\right)
$$

and the values of the amplitude of the components $j_{x}$ and $j_{z}$ of the current density are 


$$
\begin{aligned}
& j_{x}=-\frac{\omega}{\rho} \frac{16 a c^{2}}{\pi} B \sum_{m, n} \frac{1}{n} \frac{\sin \left(\frac{m \pi}{2}\right) \sin \left(\frac{n \pi}{2}\right)}{(m \pi a)^{2}+(n \pi c)^{2}} \sin \left(\frac{m \pi x}{a}\right) \cos \left(\frac{n \pi z}{c}\right) \\
& j_{z}=\frac{\omega}{\rho} \frac{16 c a^{2}}{\pi} B \sum_{m, n} \frac{1}{m} \frac{\sin \left(\frac{m \pi}{2}\right) \sin \left(\frac{n \pi}{2}\right)}{(m \pi a)^{2}+(n \pi c)^{2}} \cos \left(\frac{m \pi x}{a}\right) \sin \left(\frac{n \pi z}{c}\right)
\end{aligned}
$$

respectively. It may be noted that $j_{x}$ and $j_{z}$ are the derivative of a "current function":

$$
U(x, z)=\sum_{m, n} \frac{1}{\rho}(j \omega)\left(\frac{4 a c}{\pi}\right)^{2} \frac{B}{m n} \frac{\sin \left(\frac{m \pi}{2}\right) \sin \left(\frac{n \pi}{2}\right)}{(m \pi a)^{2}+(n \pi c)^{2}} \cos \left(\frac{m \pi x}{a}\right) \cos \left(\frac{n \pi z}{c}\right)
$$

so that $j_{x}=-\frac{\partial U(x, z)}{\partial x}$ and $j_{z}=\frac{\partial U(x, z)}{\partial z}$.

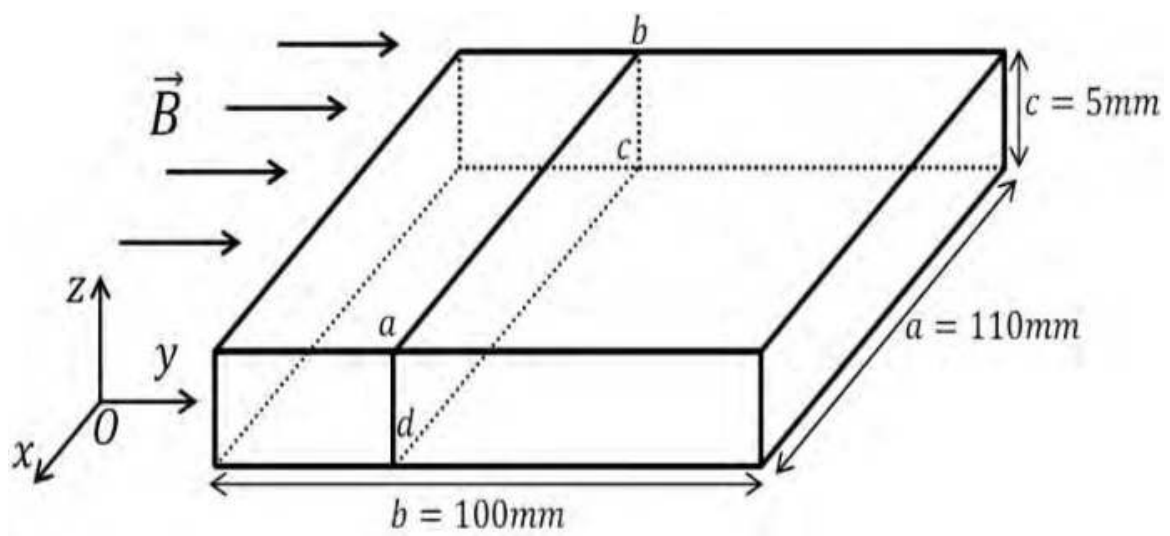

Fig. 2. Rectangular aluminium plate under consideration showing the cross section plane abcd for the evaluation of the induced currents lines

The important point is to understand that, in the case of figure $2(a=110 \mathrm{~mm}, c=5 \mathrm{~mm})$, the current density is essentially parallel to the $O x$ axis, and that this justifies the above first approximation: the current lines are parallel to $O x$ (figure 3 ).

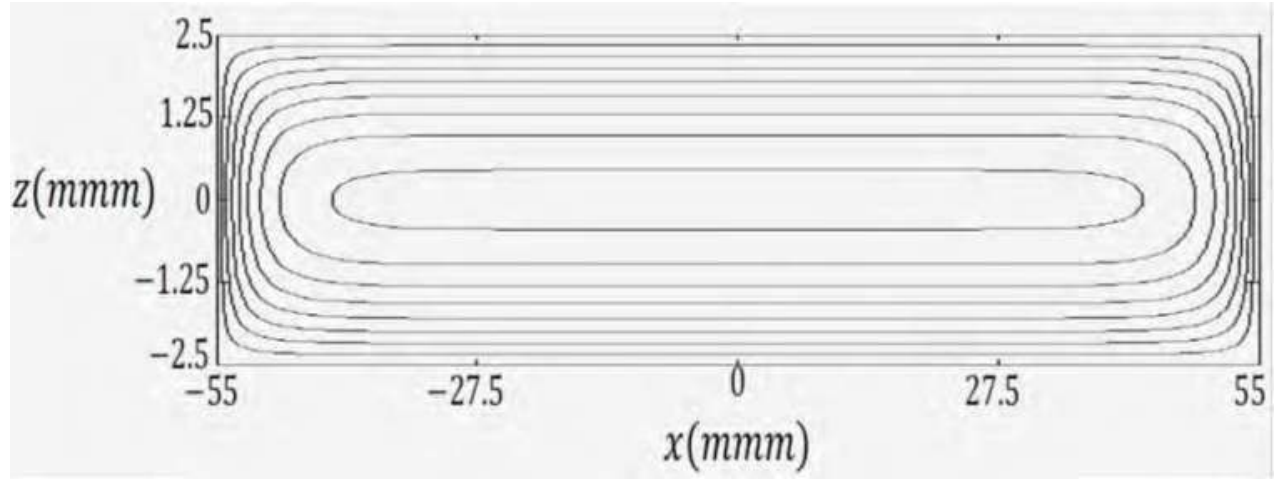

Fig. 3. Lines of induced currents for a defect free rectangular aluminium plate $(a=110 \mathrm{~mm}$, $c=5 \mathrm{~mm}$ ), the current density is essentially parallel to the $O x$ axis 


\section{Theoretical study of a rectangular aluminum plate with a calibrated crack}

Consider now the above plate, where we have made a calibrated crack, as shown in figure 4.

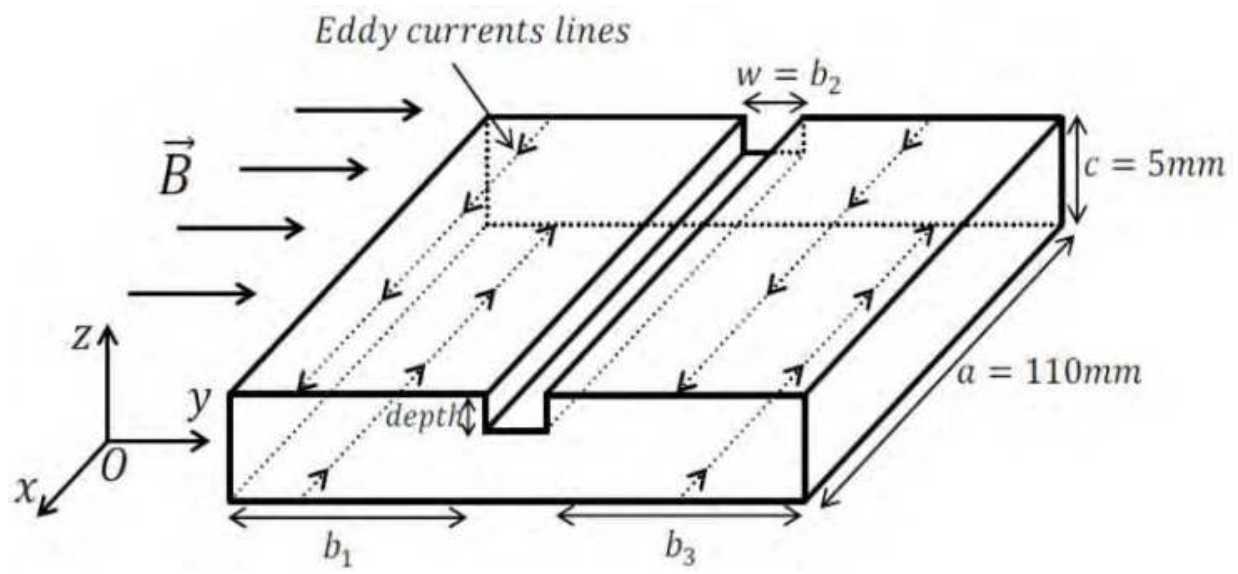

Fig. 4. Rectangular aluminium plate with a calibrated crack having a width $\left(w=b_{2}\right)$ and a depth (depth)

We analyze the induced currents produced by the alternating excitation field $\vec{B}$, considering that the plate is equivalent to 3 sane plates of widths $b_{1}, b_{2}=w$ and $b_{3}$ $\left(b_{1}+b_{2}+b_{3}=100 \mathrm{~mm}\right)$ and of thickness $c, c-$ depth and $c$ respectively. The above analysis stands for each of those three plates; the numerical analysis is straight forward, and is given below (figures 8 and 9).

\section{Experimental set-up}

In our laboratory, we have established an experimental apparatus based on Helmholtz coils arrangement for excitation, and fixed magnetic sensors (SQUID and Hall probe) (figure 5). The structure has been made mainly of Plexiglas and other non magnetic materials (brass, copper, Al, wood...) in order to avoid any magnetic noise. The samples are moved underneath the sensor by means of an $x-y$ scanning stage. The stage and the data acquisition are controlled with a LABVIEW program which is optimized to automate the entire measuring process. The sensor (SQUID or Hall probe) measures the magnetic field perpendicular to the excitation field and to the scanning direction. A lock-in amplifier has been used to achieve a synchronous detection.

The two versions of the equipment are shown in figure 6 . The sensor is located in a fixed position. In the case of the SQUID, mechanical distance between upper side of the plate and the Dewar is $0.5 \mathrm{~mm}$, and the sensor itself is $12.3 \mathrm{~mm}$ higher. In the case of the Hall probe (right part of the figure), the total distance between the plate and the active part of the sensor is $1 \mathrm{~mm}$.

The difference between the two sensors appears immediately. Indeed, the excitation field, when the SQUID is in use, is limited to $0.15 \times 10^{-4} T$ to avoid the saturation. When the Hall 
probe is in use, this limit reaches $2.45 \times 10^{-4} \mathrm{~T}$ with our generator; but it might be higher with better equipment. In both cases, the frequency is $180 \mathrm{~Hz}$. The sensitivity of the SQUID measuring sensor is $4.25 \mathrm{~V} / \mu \mathrm{T}$, and the sensitivity of the Hall sensor is $5 \mathrm{mV} / \mu \mathrm{T}$.

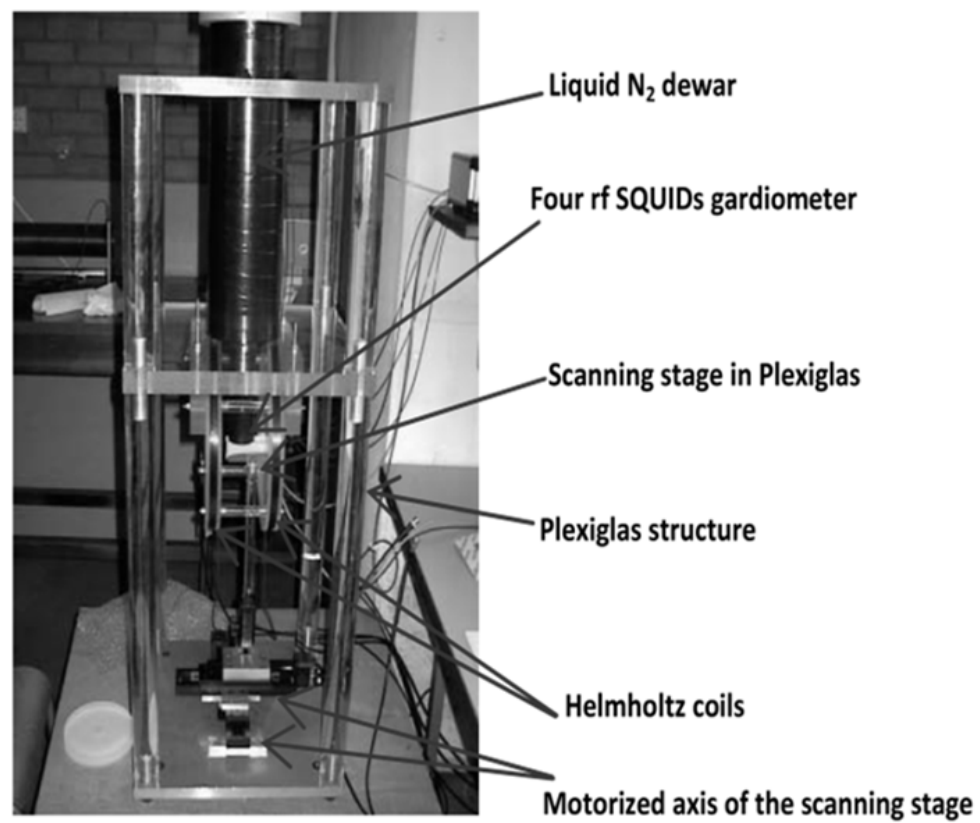

Fig. 5. High $\mathrm{T}_{\mathrm{c}}$ SQUID NDE instrumentation set-up

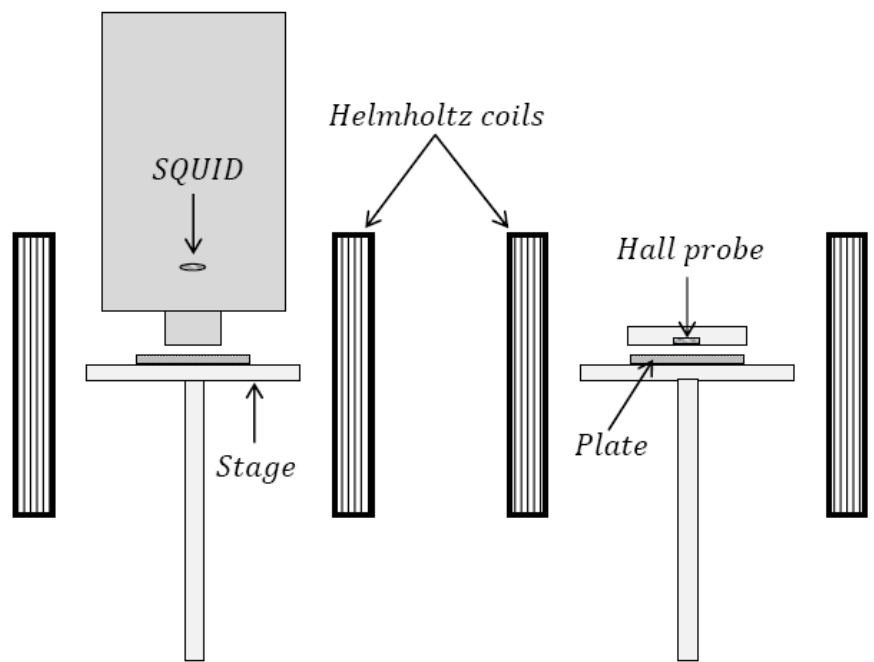

Fig. 6. The two versions of the experiment set-up: on the left is a SQUID sensor and on the right a Hall probe 


\section{Experimental verification}

Figure 7 shows the plate with a calibrated crack immersed in the uniform excitation field described above $\left(180 \mathrm{~Hz}, 0.15 \times 10^{-4} \mathrm{~T}\right.$ if we use a SQUID and $2.45 \times 10^{-4} \mathrm{~T}$ if we use a Hall probe sensor).

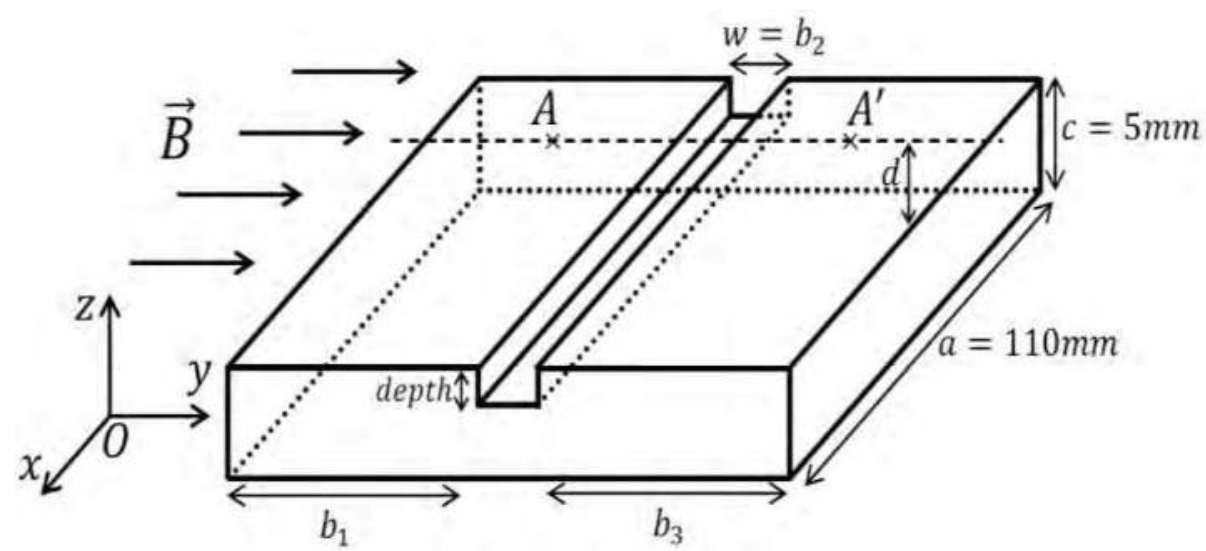

Fig. 7. Rectangular aluminium plate with a calibrated crack immersed in a uniform excitation field created by Helmholtz coils

The vertical component of the resulting induction field, measured between $A$ and $A^{\prime}$ is given at a vertical distance $d$ of $1 \mathrm{~mm}$ (measured with the Hall probe), or at a vertical distance $d$ of $12.8 \mathrm{~mm}$ (measured with the SQUID). It is clear that the computed values and the measured values tally in two very different cases (figures 8 ).

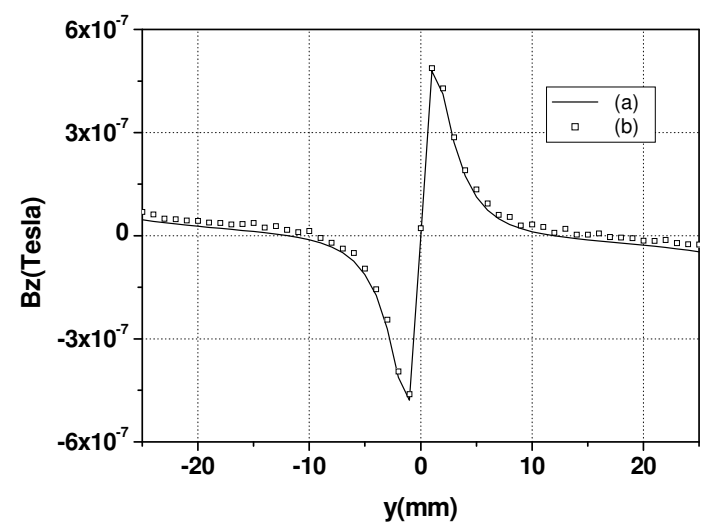

(A)

Fig. 8.A. Computed (a) and measured (b) values of $B_{z}$ between $A$ and $A$ 'in the case of a rectangular aluminium plate with a calibrated crack (width $w=1 \mathrm{~mm}$, depth $=1 \mathrm{~mm}$ ): measurement with a Hall probe at $d=1 \mathrm{~mm}$ above the plate 


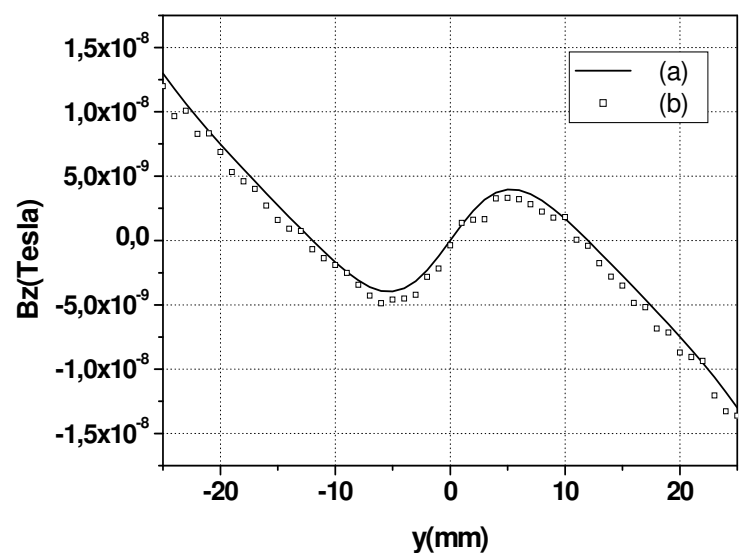

(B)

Fig. 8.B. Computed (a) and measured (b) values of $B_{z}$ between $A$ and $A$ 'in the case of a rectangular aluminium plate with a calibrated crack (width $w=1 \mathrm{~mm}$, depth $=1 \mathrm{~mm}$ ): measurement with a SQUID at $d=12.8 \mathrm{~mm}$ above the plate

Figure 9 represents the case of a defect free rectangular aluminum plate. The vertical component $B_{z}$ of the resulting induction field, measured between $A$ and $A^{\prime}$ is given at a vertical distance $d$ of $1 \mathrm{~mm}$ (measured with the Hall probe), and at a vertical distance $d$ of $12.8 \mathrm{~mm}$ (measured with the SQUID). Note that the difference between theoretical values and experimental values is so small that they cannot be distinguished.

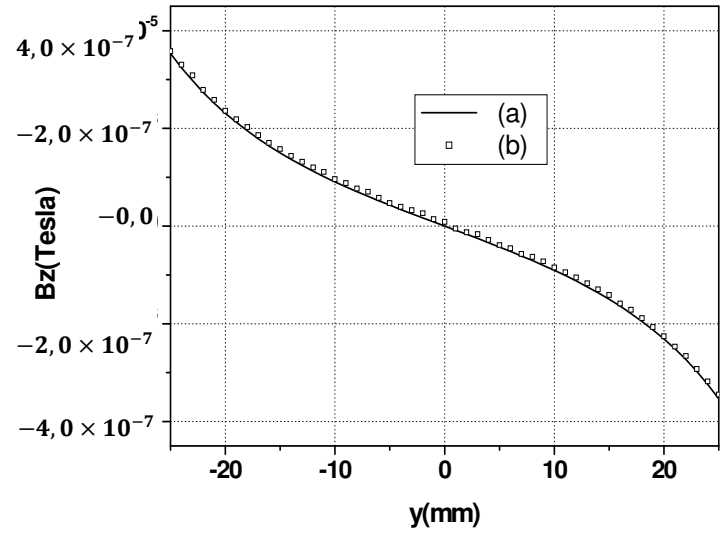

(A) 


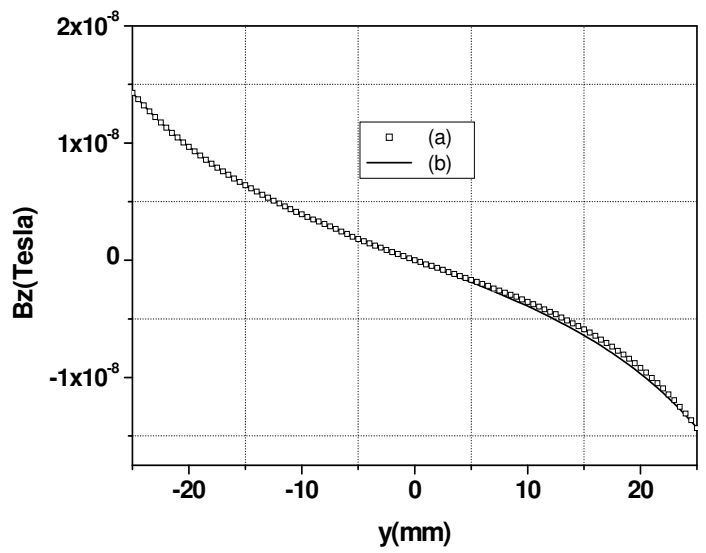

(B)

Fig. 9. Computed (a) and measured (b) values of $B_{z}$ between $A$ and $A$ 'in the case of a defect free rectangular aluminium plate: (A) measurement with a Hall probe at $d=1 \mathrm{~mm}$ above the plate, (B) measurement with a SQUID at $d=12.8 \mathrm{~mm}$ above the plate

\section{Case of a crack with zero width showing metallic dislocations}

We endeavored to repeat the same kind of experiment as above, but with a slot of zero width $\left(b_{2}=w=0 \mathrm{~mm}\right.$, depth $\left.=c\right)$ (figure 10).

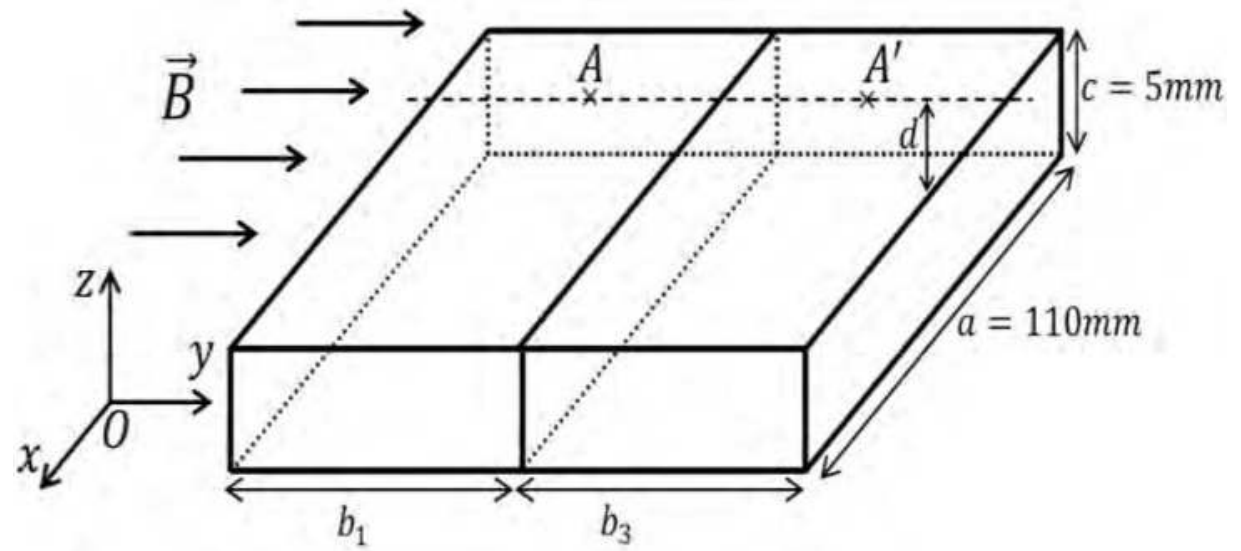

Fig. 10. Rectangular aluminium plate with a slot of zero width under consideration

We expected to find, along the line $A A^{\prime}$, a variation of the vertical component of the induced field depicted by curve (a) of figure 11, because all current lines are parallel to the $O x$ direction. In other words, we did not expect to detect the cut. We were extremely surprised to find the variation depicted by curve (b) of the same figure. In fact, we had considered that the two halves of the plate were equivalent to a homogeneous plate. What we neglected was that, when you cut a metallic plate, the cutting tool may damage the metallic microstructure 
of the plate, at least over small depth. Therefore, the intuitive representation of the situation is shown in figure 12. In this figure, the aluminum parts are themselves divided into two parts: one where the resistivity is normal $\left(\rho=5.82 \times 10^{-8} \Omega . m\right)$, and one where the resistivity is higher: $\rho^{\prime}>\rho$.

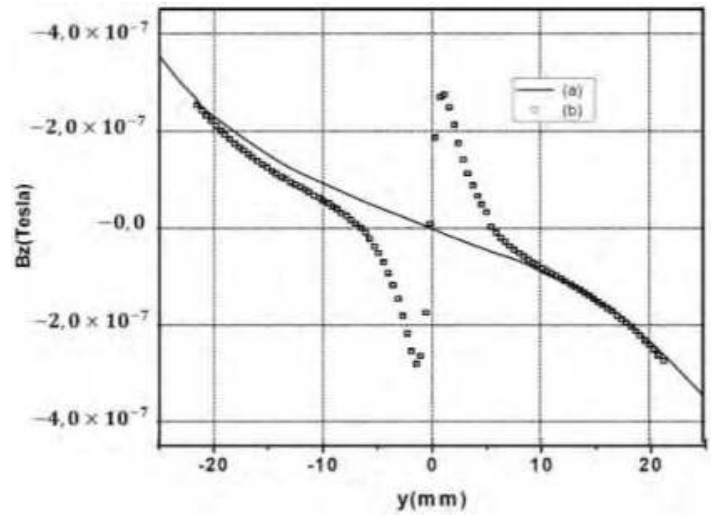

Fig. 11. Measured values of $B_{z}$, with a Hall probe at $d=1 \mathrm{~mm}$ above the plate, between $A$ and $A^{\prime}$ : (a) in the case of a defect free rectangular aluminium plate, (b) in the case of a rectangular plate with a slot of zero width

In figure 12, the thickness of the higher resistivity zone is considered $\frac{w^{\prime}}{2}$ at the edge of each half plate, so the total damaged edge area width is $w^{\prime}$.

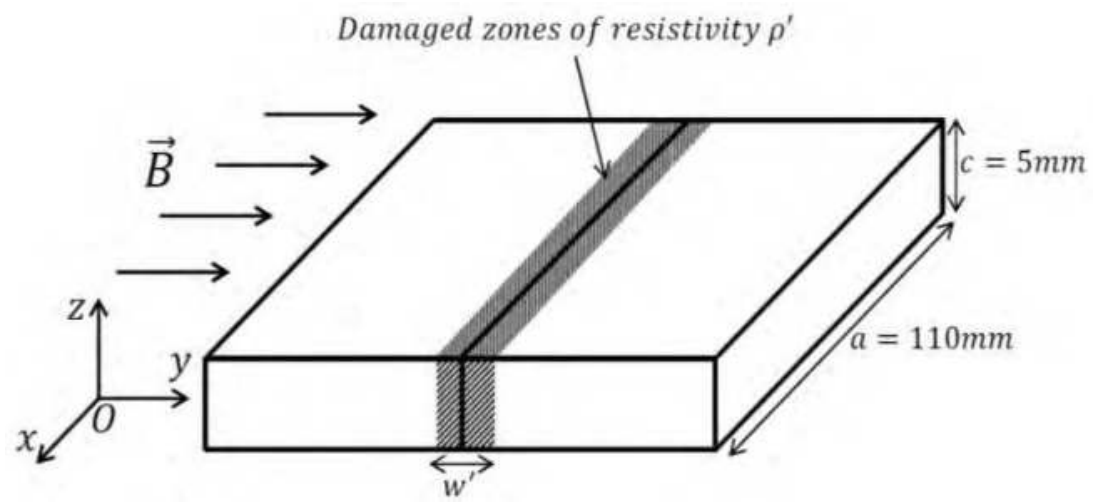

Fig. 12. Representation of the two half plates laid near each other, and of two assumed fatigued zones of resistivity $\rho^{\prime}$

Therefore, the next problem is to determine the values of $w^{\prime}$ and $\rho^{\prime}$ which account conveniently for the experimental results. This is a simple classical optimization problem. In our case, we pick at random one arbitrary value of $w^{\prime}$ and $\rho$ '. This allows to evaluate the $z$ component of the induced field. It is then necessary to define a global error $\epsilon\left(w^{\prime}, \rho^{\prime}\right)$ between the observed field, and the field corresponding to $w^{\prime}$ and $\rho^{\prime}$. Then we modify either 
$w^{\prime}$ or $\rho^{\prime}$ to decrease $\epsilon$ and repeat this process until $\epsilon$ is as small as possible. This is developed in [Poloujadoff et al., 1994] and in the reference [Bettaieb et al., 2010].

This led us to determine a best value of $\left.\rho^{\prime} \rho=5.82 \times 10^{-8} \Omega . m=1.5 \times \rho\right)$ and of $w^{\prime}\left(w^{\prime}=\right.$ $50 \mu \mathrm{m}$ ) which provided a theoretical curve of $B_{z}$ shown in figure 13 (a).

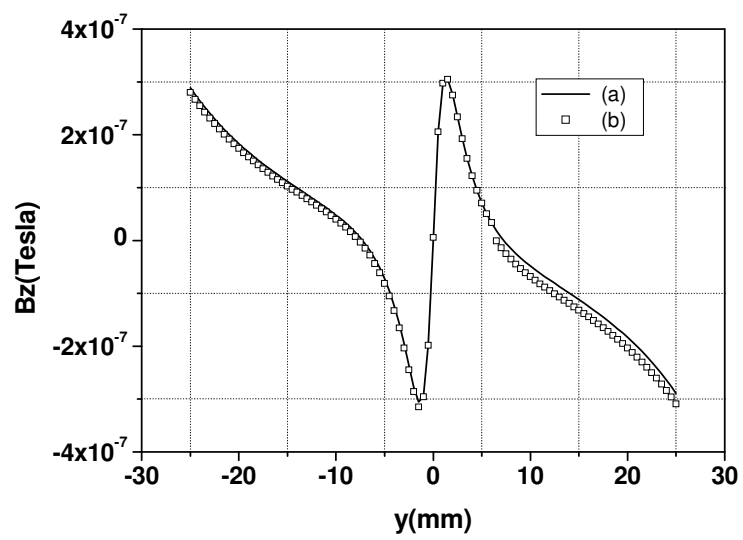

Fig. 13. (a) Theoretical curve with optimized values of $w^{\prime}=50 \mu \mathrm{m}$ and $\rho^{\prime}=1.5 \times \rho,(\mathrm{b})$ experimental curve established with a Hall sensor at $\mathrm{d}=1 \mathrm{~mm}$ above the aluminium plate in the case of a zero width crack

Since the publication of this reference, Dr. Denis GRATIAS suggested a further verification of this approach. This consisted in annealing the two half plates, then reputing the measurements. The annealing cycle is shown in figure 14.

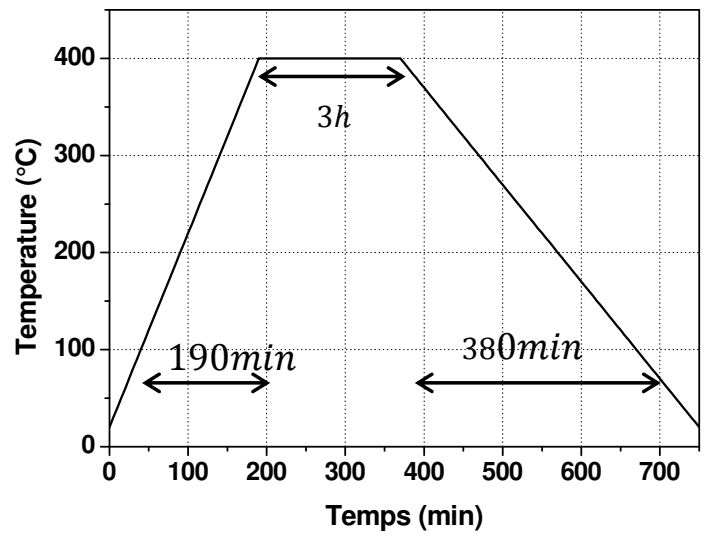

Fig. 14. Annealing cycle of the two half plates after cutting a plate

Then, we place again the two half plates in the same uniform magnetic excitation field. The curve of the vertical component of the induction field still shows a variation of $w^{\prime}$ and $\rho^{\prime}$, but much smaller than previously; this show that annealing has been very effective, but has not been long enough (figure 15). 


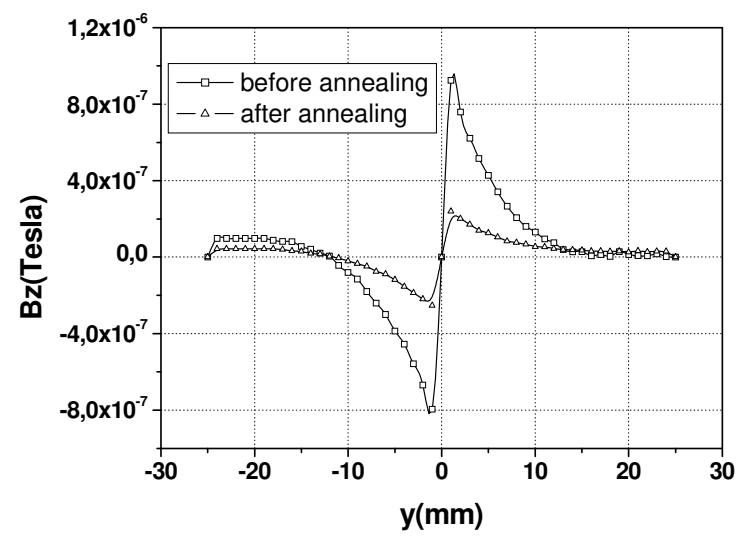

Fig. 15. Experimental curve established with a Hall sensor at $\mathrm{d}=1 \mathrm{~mm}$ above the aluminium plate in the case of a zero width crack before and after annealing

\section{Metallic dislocations created by a shock}

The above study of dislocations can be carried on by creating small damaged zones by a direct or indirect shock with a hammer (figure 16). We have already reported a preliminary study of this phenomenon [Bettaieb et al., 2010], and we report some more recent progress below.

(a)
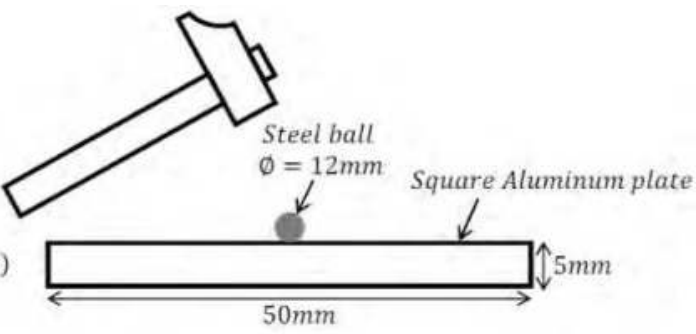

(b)

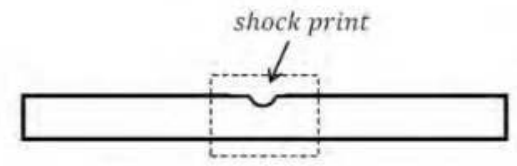

(c)

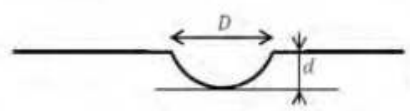

Fig. 16. (a) Creation of an impact zone by stroking a steel ball, (b) impact upon the square aluminium plate, (c) zoom of the shock print zone with modelling dimensions $(D, d)$ 
In our latest experiments, we stroke a steel ball in the middle of a square aluminum plate with a hammer. The dimensions of the damaged zones depend naturally on the radius of the steel ball and on the violence of the hammer shocks. We have considered only three cases; the depth $d$ and the diameter $D$ of some shock prints are given in the table I.

\begin{tabular}{|l|c|l|}
\hline & Dimeter $D$ & depth $d$ \\
\hline Impact 1 & $4 \mathrm{~mm}$ & $0.11 \mathrm{~mm}$ \\
\hline Impact 2 & $3 \mathrm{~mm}$ & $0.09 \mathrm{~mm}$ \\
\hline Impact 3 & $2 \mathrm{~mm}$ & $0.07 \mathrm{~mm}$ \\
\hline
\end{tabular}

Table 1. Dimensions of the damaged zones

Consider the first impact, produced near the middle of the square plate. The Aluminum plate is laid upon the stage which has been already described in the figure 6 . The excitation field being again at $180 \mathrm{~Hz}$ with an amplitude of $0.15 \times 10^{-4} \mathrm{~T}$ if we use a SQUID and $2.45 \times 10^{-4} T$ if we use a Hall probe sensor. The stage is moved as described in $\$ 4$, the variation of the vertical induction field component, measured by the Hall probe is shown in figure 17 (A). This proves that the impact may be easily detected, since the perturbation signal varies between $\pm 10^{-5} T$. It is also possible to scan a square domain $(-10 \mathrm{~mm} \leq x, y \leq+10 \mathrm{~mm})$ around the center of the impact and to represent it as in figure $17(\mathrm{~B})$.

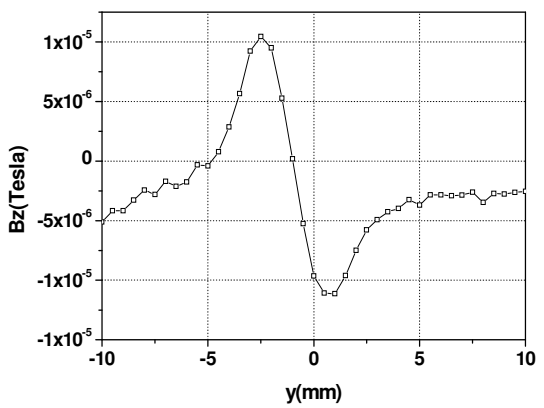

(A)

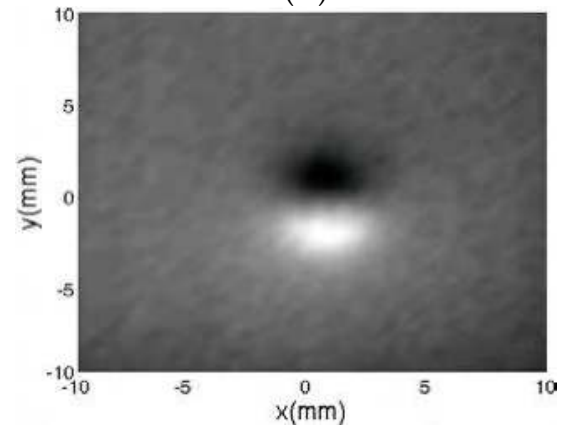

(B)

Fig. 17. (A) Measured values of $B_{z}$ along the $O y$ axis for $x=0 \mathrm{~mm}$, (B) matrix representation of the values of $B_{z}$ across a square domain $(-10 \mathrm{~mm} \leq x, y \leq+10 \mathrm{~mm})$. Measurement with a Hall probe at $\mathrm{d}=1 \mathrm{~mm}$ above the square aluminium plate $(D=4 \mathrm{~mm}, d=0.11 \mathrm{~mm})$ 
Considering the second impact, we found similar results, but the signal varies within a smaller interval $\left( \pm 0.6 \times 10^{-5} T\right)$ (see figures $18(\mathrm{~A})$ and $18(\mathrm{~B})$ ). As for the third impact, it is not really detectable.

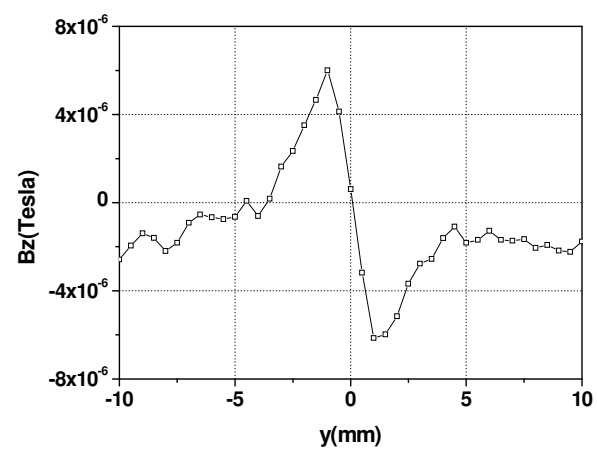

(A)

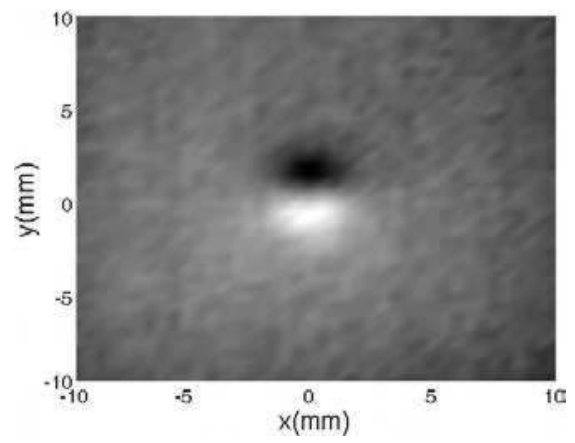

(B)

Fig. 18. (A) Measured values of $B_{z}$ along the $O y$ axis for $x=0 \mathrm{~mm}$, (B) matrix representation of the values of $B_{z}$ across a square domain $(-10 \mathrm{~mm} \leq x, y \leq+10 \mathrm{~mm})$. Measurement with a Hall probe at $\mathrm{d}=1 \mathrm{~mm}$ above the square aluminium plate $(D=3 \mathrm{~mm}, d=0.09 \mathrm{~mm})$

How may we interpret these results?

It is extremely important to consider that these results may be assigned to two different causes: the change of the shape of the aluminum square plate and/or a modification of the metallic microstructure inducing a change of the electric resistivity. To clarify this question, we have assumed that the effects of these two causes can be separated. Unfortunately, a rigorous mathematical analysis of such a structure would involve a cylindrical geometry and a uniform field, resulting in a very complicated 3D analysis. For this reason, we made the following approximations which happen to give a sufficient feeling of what happens.

This means that we first replaced the portion of a sphere, in figure 16 (c) by an empty parallelepiped with a square basis of side $w=D$ and height $h=d$ chosen to have the same volume than the portion of the half sphere (figure 19 (A)). This model accounts for the observed results (figure 19 (B)). 


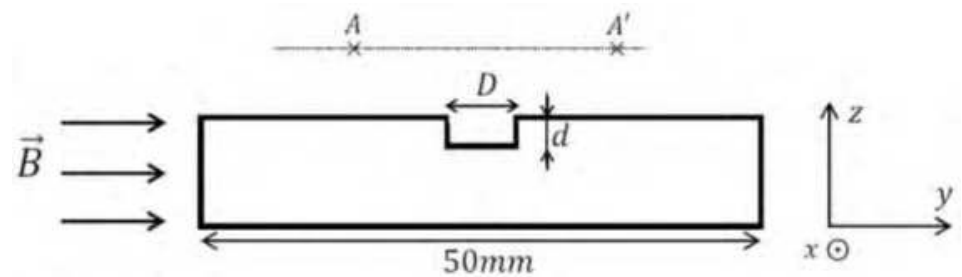

(A)

Fig. 19.A. Square aluminium plate with an empty space of same volume that the one in figure 16 (c)

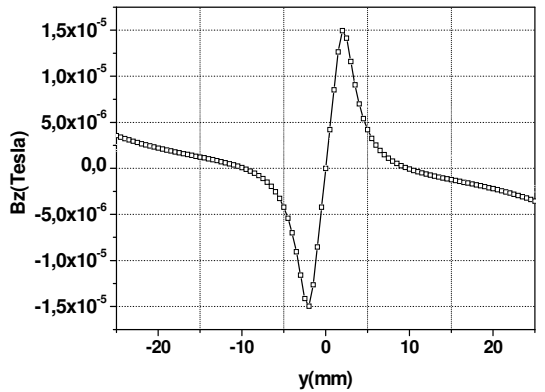

(B)

Fig. 19.B. Theoretical values of $B_{z}$ corresponding to the figure 19 (A)

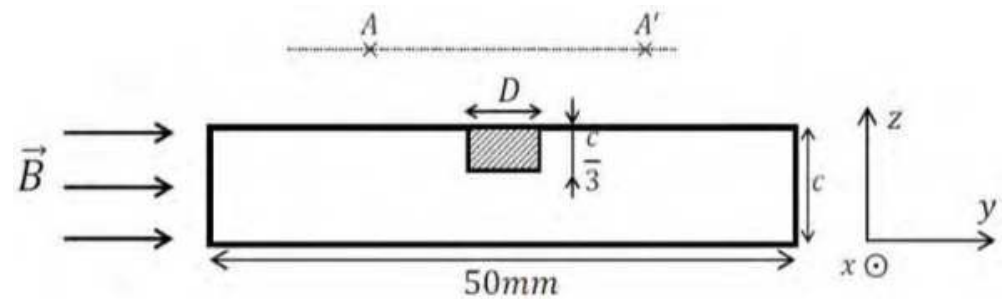

(A)

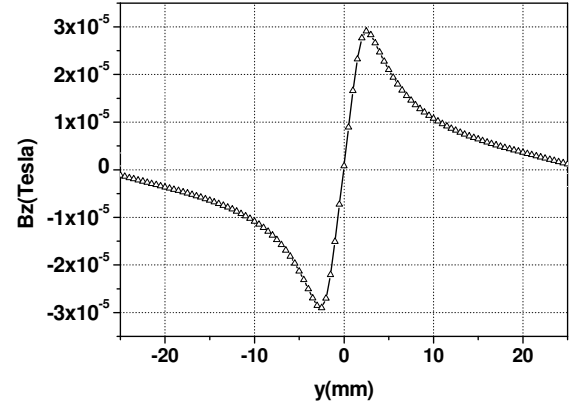

(B)

Fig. 20. (A) Square aluminium plate with an inner portion of resistivity $\rho^{\prime}>\rho,(\mathrm{B})$ theoretical values of $B_{Z}$ corresponding to the figure 20 (A). 
In a second step, we assumed that the deformation of the plate was negligible, but the metallic microstructure has been changed over a depth as large as $1 \mathrm{~mm}$. We have considered that in this region the resistivity has been changed by $10 \%$ (figure 20 (A)). This model also accounts for the experimental results (figure 20 (B)).

Therefore, this experiment and the corresponding models show that the effect of the shock is partially explained by a deformation of the square aluminum plate and partially by dislocations created in the metal over same depth. We have proved that the effects of the dislocations are much more important [Bettaieb, 2009].

\section{Metallic dislocations created by flexions}

\subsection{Experimental study}

Consider a long aluminum strip $(150 \mathrm{~mm})$ shown in figure 21 which has been bent by hand, so as to create a fatigue zone in the middle. In the already quoted previous paper [Bettaieb et al., 2010], we had shown that the fatigue of the metallic bent part could be detected. To this end, we had placed the strip in a uniform alternating horizontal field $\vec{B}$, and we have scanned its upper surface with a Hall probe. However, this first publication was limited to the feasibility of the detection.

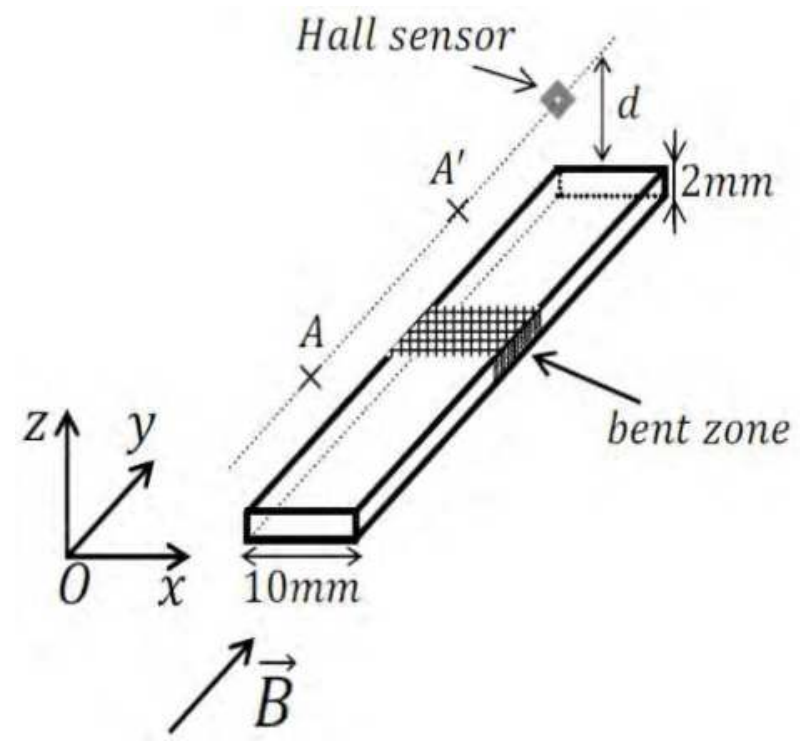

Fig. 21. Fatigued strip, the length of the damaged (bent) zone is approximately equal to $3 \mathrm{~mm}$

Since that time, we have greatly improved our experimental process, and have developed an inverse problem method for a better mathematical analysis of the results.

Our first effort has been to flatten the long strip carefully before measurement, as shown by figures 22 (figure 22 (A) is a side photo, and figure 22 (B) an upside down view of the strip during flattening). As a result, the signal obtained along the sensor track is symmetrical about its center (figure $23(\mathrm{~A})$ ). 


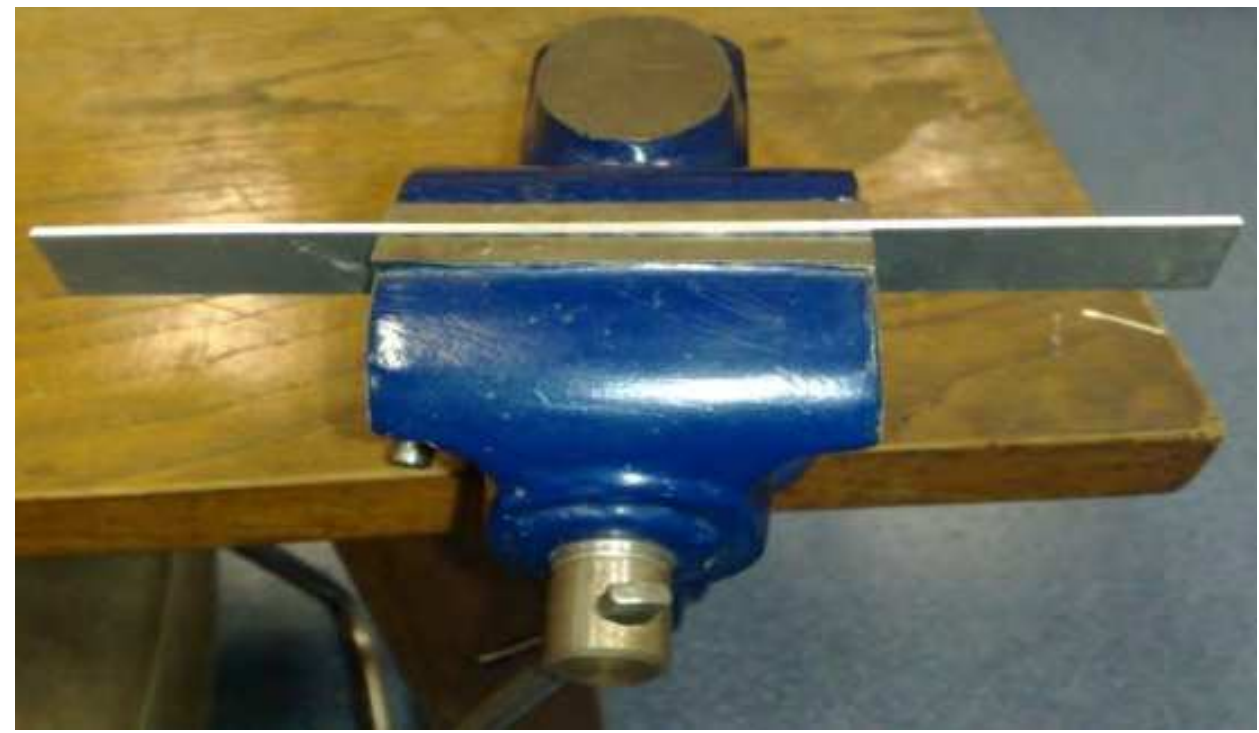

(A)

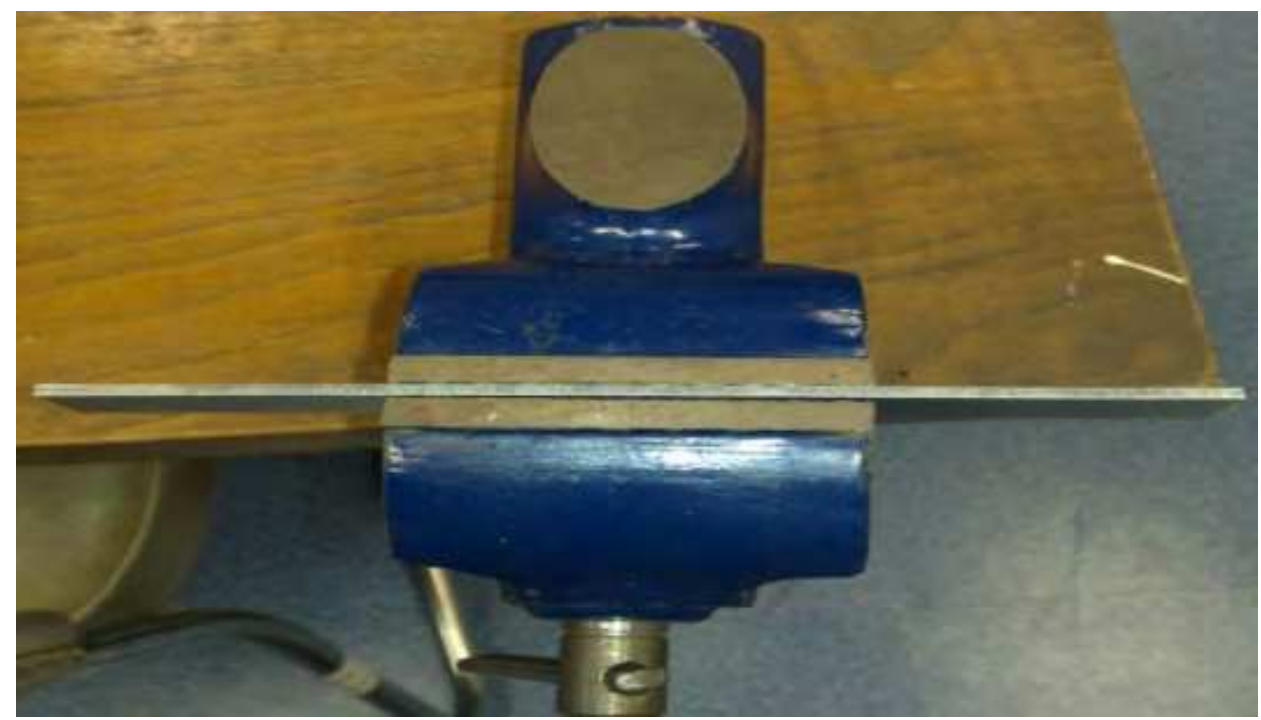

(B)

Fig. 22. Bent long strip being flattened in a shaper to eliminate the geometrical deformation before eddy current measurement (A: side view; B: upper view) 


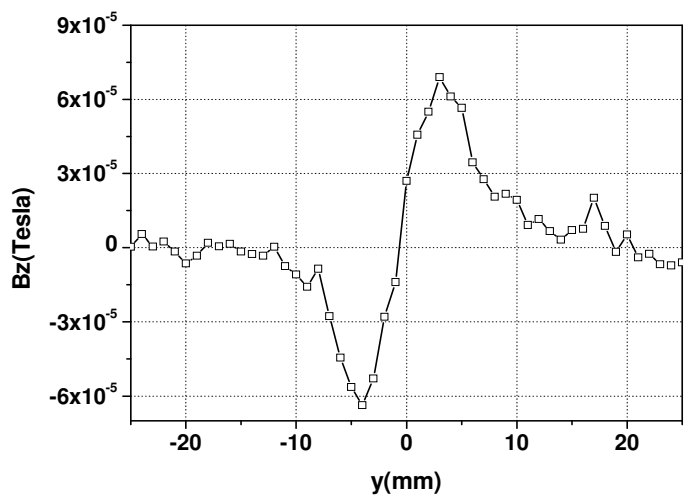

(A)

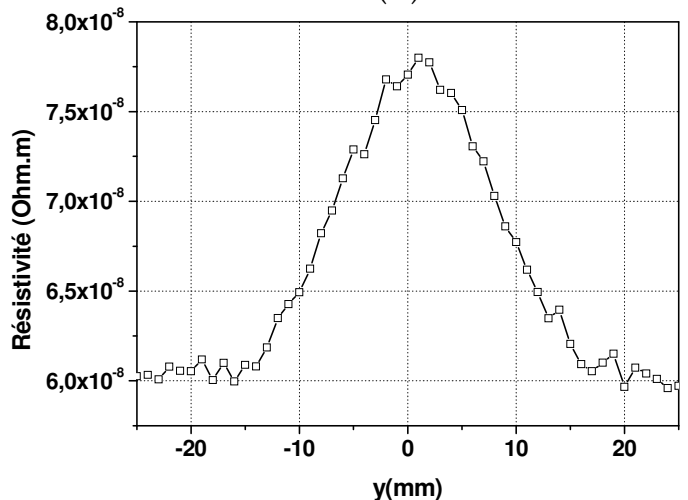

(B)

Fig. 23. (A) Values of $B_{z}$ induced by the eddy currents within a flattened fatigued long strip (measurement with a Hall sensor at $d=1 \mathrm{~mm}$ above the strip), (B) Corresponding values of the estimated local resistivity along the damaged zone using the inverse solution

\subsection{Theoretical evaluation of resistivity along the strip}

The principle of the inverse problem is well known. The relationship between the induction field and the induced currents in the long strip is linear, the coefficients being the conductivity. Simultaneously, the relationship between the strip induced current and the outside induced induction field is also linear. Therefore, if the exciting induction field and the induced induction field are known, the resistivity can be determined [Bettaieb et al., 2010]. It is what has been done to estimate the resistivity (figure 23 (B)) along the strip. This latter result clearly shows the effect of the fatigue where the strip has been bent.

\section{Conclusion}

In this very original chapter, we have shown that a methodology generally used to detect cracks can be also used to measure the effects of a mechanical fatigue created by cutting tools, impact shocks or flexions. The natural continuation should be to study quantitatively 
most of the microstructure properties which influence the resistivity of the alloy. These include irradiations defects and phase transitions. We would certainly welcome collaboration offers from colleagues.

\section{Acknowledgment}

We would like to thank warmly Dr. Denis GRATIAS from ONERA/CNRS for several useful advices and discussions notably related to the nature of dislocations. He also suggested the check of our ideas by the annealing procedure.

\section{References}

Bettaieb, L. (2009). Contributions to Non Destructive Evaluation of amagnetic metlas using eddy currents. Phd Thesis at University of Pierre and Marie Curie (UPMC), in French, (September 2009).

Bettaieb, L.; Kokabi, H.; Poloujadoff, M.; Sentz, A. \& Krause, H. J. (2008). Non Destructive Testing (NDT) With High Tc RF SQUID. Journal of Physics: Conference Serie 97, (March 2008), 6pp.

Bettaieb, L.; Kokabi, H.; Poloujadoff, M.; Sentz, A.; Krause, H. J. \& Coillot, C. (2010). Comparison of the use of SQUID an Hall effect sensors in NDE. Materials Evaluation, Vol.68, No.5, (2010), pp. 535-54.

Bettaieb, L.; Kokabi, H.; Poloujadoff, M.; Sentz, A. \& Tcharkhtchi, C. (2010). Fatigue and/or crack detection in NDE. Nondestructive Testing and Evaluation, Vol.25, No.1, ( March 2010), pp. 13-24.

Bettaieb, L.; Kokabi, H.; Poloujadoff, M.; Sentz, A. \& Krause, H. J. (2009). Analysis of some Nondestructive Evaluation Experiments Using Eddy Currents. Research in Nondestructive Evaluation, Vol.20, No.3, (July 2009), pp. 159-177.

Poloujadoff, M.; Christaki, E. \& Bergman, C. (1994). Univariant search: An opportunity to identify and solve conflict problems in optimization. IEEE trans. Energy Conver., EC(9), (December 1994), pp. 659-664. 
NONDESTRUCTIVE

TESTING METHODS

AND NEW

APPLICATIONS

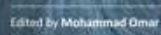

\section{Nondestructive Testing Methods and New Applications}

Edited by Dr. Mohammad Omar

ISBN 978-953-51-0108-6

Hard cover, 264 pages

Publisher InTech

Published online 02, March, 2012

Published in print edition March, 2012

Nondestructive testing enables scientists and engineers to evaluate the integrity of their structures and the properties of their materials or components non-intrusively, and in some instances in real-time fashion. Applying the Nondestructive techniques and modalities offers valuable savings and guarantees the quality of engineered systems and products. This technology can be employed through different modalities that include contact methods such as ultrasonic, eddy current, magnetic particles, and liquid penetrant, in addition to contact-less methods such as in thermography, radiography, and shearography. This book seeks to introduce some of the Nondestructive testing methods from its theoretical fundamentals to its specific applications.

Additionally, the text contains several novel implementations of such techniques in different fields, including the assessment of civil structures (concrete) to its application in medicine.

\section{How to reference}

In order to correctly reference this scholarly work, feel free to copy and paste the following:

Bettaieb Laroussi, Kokabi Hamid and Poloujadoff Michel (2012). Study of Metallic Dislocations by Methods of Non Destructive Evaluation Using Eddy Currents, Nondestructive Testing Methods and New Applications, Dr. Mohammad Omar (Ed.), ISBN: 978-953-51-0108-6, InTech, Available from:

http://www.intechopen.com/books/nondestructive-testing-methods-and-new-applications/study-of-metallicdislocations-by-methods-of-non-destructive-evaluation-using-eddy-currents

\section{INTECH}

open science | open minds

\section{InTech Europe}

University Campus STeP Ri

Slavka Krautzeka 83/A

51000 Rijeka, Croatia

Phone: +385 (51) 770447

Fax: +385 (51) 686166

www.intechopen.com

\section{InTech China}

Unit 405, Office Block, Hotel Equatorial Shanghai

No.65, Yan An Road (West), Shanghai, 200040, China

中国上海市延安西路65号上海国际贵都大饭店办公楼 405 单元

Phone: +86-21-62489820

Fax: $+86-21-62489821$ 
(C) 2012 The Author(s). Licensee IntechOpen. This is an open access article distributed under the terms of the Creative Commons Attribution 3.0 License, which permits unrestricted use, distribution, and reproduction in any medium, provided the original work is properly cited. 